\title{
Análisis de los cuellos de botella en la logística internacional de las Pymes de confecciones en Colombia
}

\author{
Competitive logistics in exporting SMEs in Colombia: analysis of \\ bottlenecks and improvement proposals
}

\author{
Egna Avendaño Cardenas* y Harold Silva Guerra**
}

\section{RESUMEN}

El objetivo de esta investigación fue analizar los cuellos de botella del proceso logístico internacional - desde el proveedor de la materia prima hasta la entrega del consumidor final- que afectan la competitividad de las Pymes de confecciones de Colombia en el mercado internacional. El artículo se sustenta en tres marcos teóricos, la teoría de restricciones (Goldratt, 1994), la teoría de competitividad (Porter, 1997) y la trilogía logística (Bloch, 2011a), es un tipo de investigación descriptiva, con un análisis de datos cuantitativos y cualitativos. Para la recolección de información se aplicaron encuestas en dos empresas líderes de confecciones en Colombia: Los Vestidos e Industrias Goriz, así mismo se encuestaron 9 expertos en Pymes de confecciones del programa expopyme de Procolombia. Se concluyó que es importante la coordinación de la logística interna de la empresa con la logística de exportaciones, así mismo se identificaron restricciones en la cadena logística como la capacidad productiva, el cumplimiento de tiempos de producción, la poca claridad en el costo del producto final, la falta de estudios de mercado en los países destino, el transporte de mercancías, el desconocimiento de documentación por parte del empresario, la falta de liquidez para aplicar los requerimientos internacionales y la falta de trabajo conjunto de las pymes con el estado para el mejoramiento de condiciones competitivas.

Palabras claves: Logística empresarial, logística exportaciones, cadena de suministro, competitividad, cuellos de botella, pymes y comercio exterior.

\footnotetext{
* Master en Gestión estratégica del comercio exterior y la integración. UBA. Profesora tiempo completo Escuela de Negocios Universidad del Norte, Colombia. Correo electrónico: egnaa@ uninorte.edu.co

** $\mathrm{PhD}$ in Management. Profesor investigador de tiempo completo de la Escuela de Negocios Universidad del Norte, Colombia. Miembro del Grupo de Investigación en Marketing (GIM). Correo electrónico: hsilva@ uninorte.edu.co
} 


\begin{abstract}
The objective of this research was to analyze the effects of bottlenecks in the logistics process - from the supplier of the raw material to the delivery of the final consumer on the competitiveness of Colombian garment exporting SMEs. The article is based on three theoretical frameworks, the theory of restrictions (Goldratt, 1994), the theory of competitiveness (Porter, 1997) and the logistic trilogy (Bloch, 2011a), is a type of descriptive research, with a data analysis quantitative and qualitative .For the collection of information, surveys were used to 7 experts in clothing SMEs, especially the expopyme program of Procolombia carried out in 2007 and statistical data of the World Bank (World Bank, 2016), ANDI (National Association of Industrialists, 2016) and of Trade Map (International Trade Center, 2017).It was concluded that there are two factors that influence competitiveness: on the one hand internal logistic processes of the company and those of export, are analyzed separately to give clarity to the handling of the procedures, but this little coordination of the logistic process in its entirety decreases competitiveness. On the other hand, bottlenecks in the logistics process require the joint effort between SMEs and government management. That is why the article hopes to expose the logistical constraints in a joint way to propose solutions to SMEs that wish to internationalize.
\end{abstract}

Keywords: Business logistics, exports logistics, supply chain, competitiveness, bottlenecks, SMEs and foreign trade.

\title{
Introducción
}

Las pequeñas y medianas empresas- Pymes- son parte del crecimiento económico de los países, siendo así necesario mejorar sus condiciones internas para afrontarse a los retos actuales (Montoya \& Montoya, 2010), y de esta manera crear Pymes con altos estándares que se enfrenten con el mercado internacional de manera exitosa.

En Colombia la clasificación de empresas establecida en el artículo $2^{\circ}$ de la Ley 905 de 2004, son grandes, medianas y pequeñas y microempresas. Para el 2.016 se registraron en el sector textil-confección 725 empresas de las cuales el 24,0\% corresponde a grandes empresas, el $56,1 \%$ a medianas, el $19,9 \%$ a pequeñas y microempresas (Superintendencia de Sociedades, 2016). 
Análisis de los cuellos de botella en la logística internacional de las Pymes de confecciones en Colombia

Aunque aproximadamente el 70\% son Pymes, para el caso concreto del sector de confecciones la participación en ingresos operacionales se concentra en las grandes empresas como lo muestra el estudio de la Superintendencia de sociedades del año 2016, donde se analizaron dos grupos; el primero con 121 empresas del sector textilconfecciones de las cuales 96 empresas grandes tuvieron una participación del 97\% y las Pymes 3\% sobre el total de los ingresos percibidos por la operación en el $2016^{1}$. El segundo grupo 604 empresas estudiadas ${ }^{2}$ las empresas grandes tienen una participación del $52,7 \%$ en el total de los ingresos operacionales, mientras que las medianas participan con el $41,9 \%$ y las más pequeñas y micros aportan el $5,4 \%$.Las empresas líderes en confección de prendas para el 2016 son las siguientes: Crystal S.A.S, STF GROUP SA, Permoda LTDA, Tennis S.A y Leonisa S.A. (Superintendencia de Sociedades , 2016).

Con respecto a la participación del sector de confecciones en Colombia para el año 2.016, esté contribuyó en el $8,8 \%$ del PIB de la industria manufacturera(Superintendencia de Sociedades , 2016), disminuyó su producción en 2,3\% en Hilatura, tejeduría y acabado de productos textiles y un 5,9\% en confección de prendas de vestir y bajaron las ventas de confecciones un 1,9\% Departamento Administrativo Nacional de Estadística (DANE, 2016).

En el comercio exterior las exportaciones del sector de confecciones presentó un descenso en todos los subsectores, por ejemplo en preparación e hilatura de fibras textiles se presentó una variación negativa del $15,3 \%$, en fabricación de tejidos de punto un $-19,1 \%$ y fabricación de prendas de vestir un -17,3\%(DANE, 2016).

Así mismo en las importaciones mostraron un comportamiento decreciente en valores CIF en la mayoría de subsectores. La fabricación de prendas de vestir, tuvo

\footnotetext{
${ }^{1}$ Se trabajó con información financiera de una muestra de 121 empresas con diferente naturaleza jurídica, tamaño y operación para los años 2015 y 2016 y que se clasificó en los siguientes 3 subsectores teniendo cuenta el tipo de negocio y su cadena de valor: (i) fabricación de productos textiles5 con 35 empresas, (ii) confección de prendas6 con 37 y (iii) comercio con 49 empresas

2 Se estudió la situación financiera de una muestra de 604 empresas con diferente naturaleza jurídica, tamaño y operación, para los años 2015 a 2016 y La Superintendencia clasificó los siguientes 3 subsectores teniendo cuenta el tipo de negocio y su cadena de valor: (i) fabricación de productos textiles 8 con 108 empresas, (ii) confección de prendas con 243 y (iii) comercio 10 .
} 


\section{Egna Avendaño Cardenas y Harold Silva Guerra}

Telos Vol. 20, No. 3 (2018). 510-536

la cifra más negativa de todos los subsectores con el $22 \%$, seguido de fabricación de otros productos textiles con el $21,5 \%$, fabricación de tejidos y artículos de punto ganchillo con el $11,1 \%$ y tejedura de productos textiles que registró $4,6 \%$ menos. Por su parte, el de preparación e hilatura de fibras textiles registró un aumento del 6,9\%(DANE, 2016).

Como se observa en los datos el sector textil y confecciones en Colombia, está en un proceso de transición y adaptación en la aplicación de tratados de libre comercio $\mathrm{y}$ en las estrategias para afrontar la competencia internacional, especialmente de China. Para lograr lo cual su paso más importante es lograr una logística eficaz al interior de la empresa y en su proceso externo de exportaciones mejoramiento de infraestructura y servicios prestados para obtener costos competitivos (Guasch, 2011).

Con el fin de analizar los cuellos de botella en el proceso logístico para mejorar la eficiencia de las pymes de confecciones, el enfoque del artículo se respalda en la teoría de las restricciones que afirma que las empresas tendrían ganancias ilimitadas si se eliminaran sus cuellos de botella(Goldratt, 1990), se complementa con Michael Porter en su teoría de ventaja competitiva (Porter, 1997), la cual expone la competitividad de una empresa se da por tres tipos básicos de ventaja competitiva, liderazgo de costos, diferenciación y enfoque y con la trilogía logística de Roberto Bloch (Bloch, 2011a), que expone que existen tres elementos: rapidez, eficiencia y seguridad, que combinados adecuadamente en la cadena logística logra una mayor competitividad de las empresas exportadoras.

La metodología utilizada fue el uso encuestas a dos empresas y 9 expertos y el análisis estadístico, las encuestas a empresas presentaron dificultades, en un inicio se esperaban realizar 20, pero las empresas exportadoras de confecciones en su mayoría son reservadas con la información productiva, sin embargo, se obtuvieron de dos reconocidas empresas de confecciones Industrias Goriz y los vestidos. Para respaldar los conceptos, después de realizar las encuestas a los empresarios, se definió 
Análisis de los cuellos de botella en la logística internacional de las Pymes de confecciones en Colombia

la necesidad de conocer la visión de consultores expertos que trabajan en pymes y que habían asesorado pymes de confecciones,

El objetivo de esta investigación fue analizar los cuellos de botella del proceso logístico internacional - desde el proveedor de la materia prima hasta la entrega del consumidor final- que afectan la competitividad de las Pymes de confecciones de Colombia en el mercado internacional, para lo cual se divide en las siguientes partes: La primera parte hace referencia a los conceptos de logística interna y de exportaciones, mostrando la importancia de trabajar los conceptos en conjunto y no por separado, la segunda parte analiza la teoría de las restricciones y la teoría de competitividad aplicándolas al sector de confecciones de Colombia y la tercera parte analiza las restricciones logísticas al interior de las empresas y en el procedimiento de exportaciones, resultado de las encuestas realizadas.

\section{Conceptualización de la logística}

La logística es entendida como:

"El conjunto de todas las actividades relacionadas con el flujo de materiales desde el punto proveedor hasta el punto consumidor, contempla además de las actividades materiales aquellas mediante las que se planifica, organiza, regula y controla dicho flujo material (dirección) de forma eficiente entendiéndose por eficiente llegar al punto consumidor con la cantidad y calidad requerida en el momento y lugar demandado con el menor costo posible". (Goméz y Acevedo, 2000, p. 6)

Con respecto al concepto de logística empresarial y de logística internacional, se diferencian en el ámbito de actuación. Por un lado, como lo afirma Guash (2011), la logística empresarial incluye las actividades internas de la empresa relacionadas con el flujo y transformación de materiales, información y dinero, desde la materia primaextracción- hasta el usuario final. Los elementos que se tienen en cuenta en el proceso logístico al interior de la empresa son los proveedores, los centros de producción, los canales de distribución y los clientes. (Carro \& González, 2008). 
Por otro lado, la logística internacional coordina actividades desde la entrega de mercadería en la empresa hasta su destino en otro país , es así como la logística internacional se define como: "la movilidad de productos o servicios desde un país determinado hacia otros países" (Bloch, 2011a, p.10), e incluye las operaciones necesarias requeridas para lograr el traslado físico desde el local del exportador de las mercancías hasta el local del importador en las condiciones pactadas, en los tiempos requeridos y con el costo adecuado (Antún, 2004)

Estos conceptos fueron identificados por los entrevistados de la siguiente manera:

Figura1. Conceptos de logística empresarial y logística de exportaciones
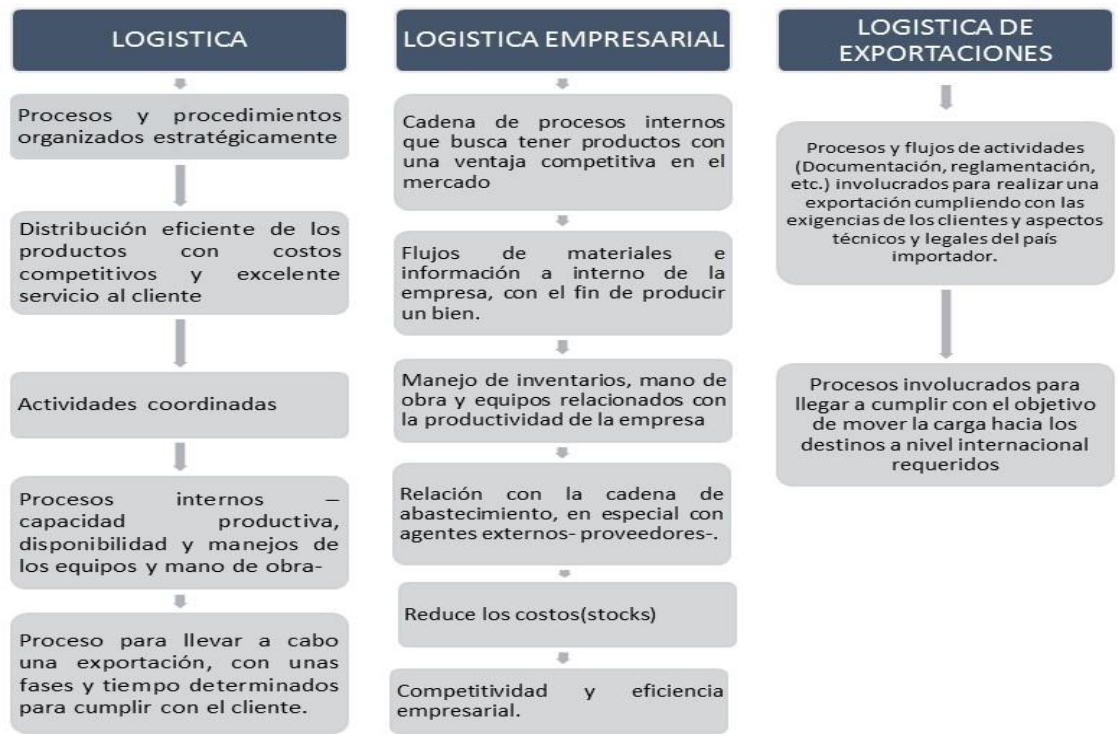

Fuente. Elaboración propia.

Teniendo en cuenta lo anterior en el siguiente gráfico se explican los elementos de la logística empresarial, los cuales el gerente tiene el control directo y debe asegurar su eficiencia para minimizar el riesgo en las exportaciones. (Long, 2006). 
Análisis de los cuellos de botella en la logística internacional de las Pymes de confecciones en Colombia

Figura 2. Cadena Logística Empresarial
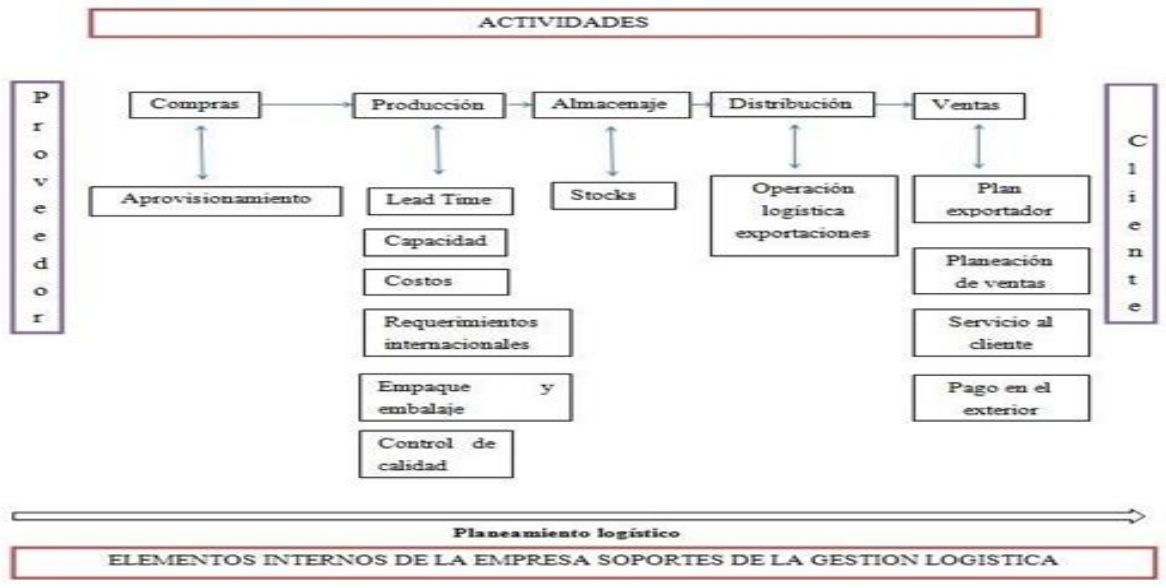

Fuente. Adaptado de la Cámara de Comercio de Bogotá (2017)

Con relación a la logística internacional, es parte del proceso de distribución (Long, 2006), como se muestra en el anterior gráfico e incluye elementos más específicos como se detalla a continuación:

Figura 3. Cadena logística exportaciones

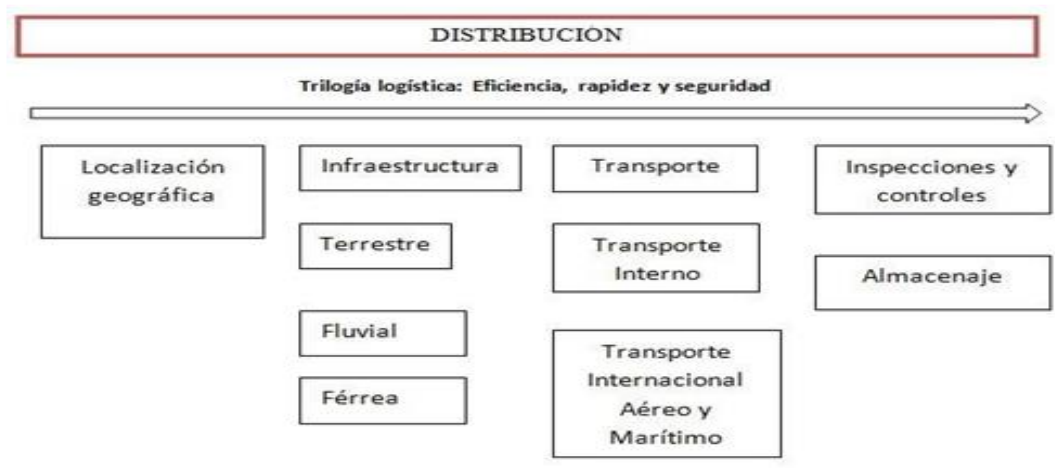

Flujo de Información $y$ documentos

ELEMENTOS EXTERNOS EMPRESA QUE INFLUYEN EN LA GESTION LOGISITCA

Fuente. Elaboración Propia, basado en conceptos de Long (2006) y Bloch (2011a) 
El anterior gráfico incluye el proceso de cómo lograr la eficiencia logística en la empresa en su proceso de exportación que conforman una trilogía de valores: Rapidez, eficiencia y seguridad en la cadena logística. (Bloch, 2011a). Rapidez entendida como llegar en el plazo acordado al destino, eficiencia como el cumplimiento del objetivo de entrega del bien al menor costo posible y seguridad que el bien llegue a destino en óptimas condiciones. (Bloch, 2011b). Lo que se busca es que la cadena logística logre brindar una adecuada combinación en la aplicación de los tres valores mencionados, lo que traerá como consecuencia una mayor competitividad para la empresa.

De esta manera, el empresario debería tener conocimiento del proceso logístico desde su proveedor de materia prima - País A- hasta la entrega a su cliente final- País B-, buscando que el producto llegue en la cantidad y calidad requerida en el momento y lugar demandado con el menor costo posible y es por esta razón que la logística empresarial y la internacional deben estar coordinadas en todos sus procedimientos.

Es por esta razón que la industria debe buscar el control de toda la cadena logística como factor de competitividad. (Acosta \& Guerrero López, 2005) y las pymes de Colombia necesitan fortalecer su capacidad con el fin de responder rápidamente a los ajustes de las situaciones macroeconómicas, así mismo como se debe tecnificar y mejorar la calidad (Jian, Xiangdong, \& Zhihui, 2008). Cuando el proceso logístico empresarial se hace eficiente, logra que la logística exportadora dé un mejor resultado para el cliente final. (Guasch, 2011).

Según la Cámara de Comercio de Bogotá (2017)

"hay que trabajar en la articulación de los distintos eslabones de la cadena. Este imperativo no solo abarca la necesidad de avanzar en procesos de asociación, como mecanismo de los pequeños empresarios que caracterizan el sector, puedan hacer frente a la gran demanda internacional, sino además la conveniencia de generar una mayor integración vertical que permita alcanzar una mayor agregación de valor" (p. 10). 
Análisis de los cuellos de botella en la logística internacional de las Pymes de confecciones en Colombia

Por su lado los entrevistados afirman que hay conectividad entre la logística interna y la logística de exportación por las siguientes razones:

1. Responde a las necesidades del cliente final, debido a la necesidad de conocer la disponibilidad de materia prima, de los equipos, de la mano de obra y de la capacidad instalada para responder con los clientes en el exterior.

2.Buscan cumplir los objetivos de la empresa: Por ejemplo, si la empresa busca internacionalizarse, debe enfocar sus procesos al cumplimiento de normatividades y requerimientos del mercado al que piensa llegar -empaques, calidad, certificados- y estandarizar sus procesos productivos con el fin de cumplir con los tiempos y calidad.

Para el caso de confecciones esta conectividad se puede observar en el siguiente gráfico, desde el insumo primario hasta empaque y embalaje nos indica lo perteneciente al proceso logístico empresarial y del transporte interno hasta la evaluación de la gestión logística de exportaciones.

Figura 4. Proceso logístico de exportación de confecciones 


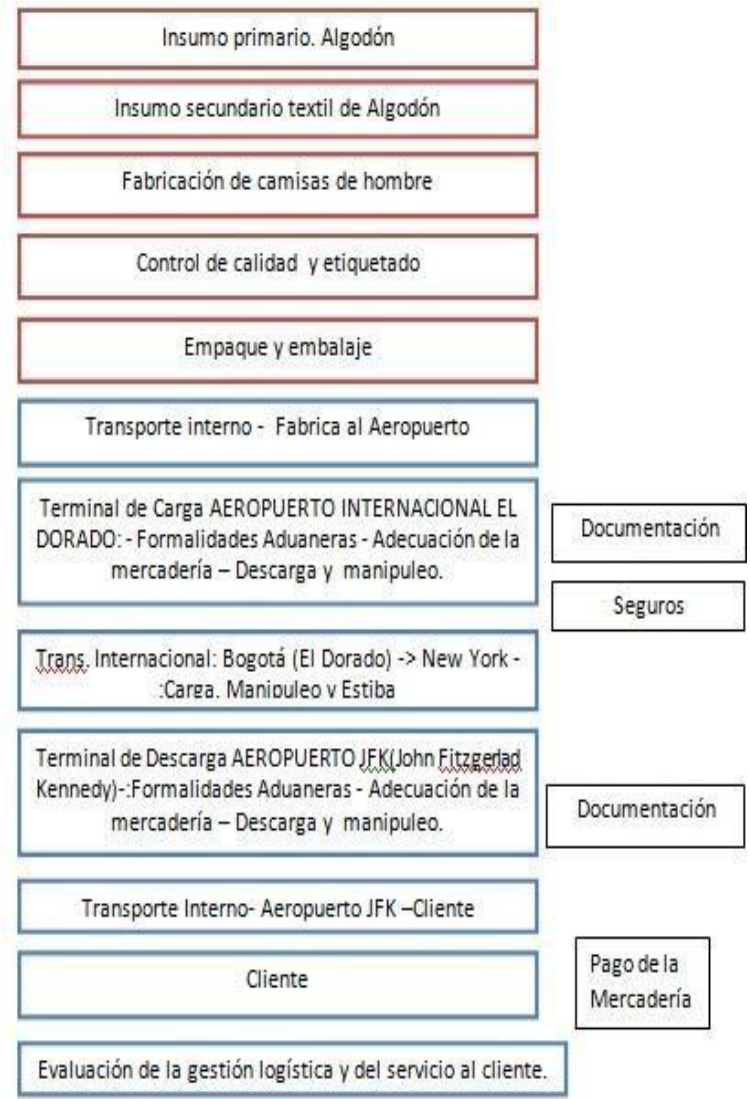

Fuente: Elaboración propia.

Es así como, para llevar a cabo una logística exitosa es necesario planear, implementar, y controlar efectiva y eficientemente las actividades básicas de la empresa como: compras, producción, almacenaje, distribución y ventas. (Goméz \& Acevedo, 2000), es decir estas actividades deben ser tratadas con mayor cuidado, para evitar las restricciones y mejorar su competitividad especialmente en costos, como se analiza en la teoría de las restricciones y la teoría de ventaja competitiva. 
Análisis de los cuellos de botella en la logística internacional de las Pymes de confecciones en Colombia

\section{Teoría de las restricciones y la teoría de ventaja competitiva.}

La Teoría de las Restricciones fue descrita por primera vez por Eli Goldratt al principio de los $80 \mathrm{y}$ desde entonces, ha sido ampliamente utilizada en la industria. La teoría enfatiza que la principal razón para que la empresa no tenga ganancias ilimitadas es por los llamados factores limitantes, denominados por el autor como restricciones o "cuellos de botella" (Goldratt, 1994). Por supuesto, las restricciones pueden ser un individuo, un equipo, una pieza de un aparato o una política local, o la ausencia de alguna herramienta o pieza de algún aparato.

De esta manera, la Teoría de las Restricciones (TOC - Theory of Constraints), demuestra que en toda empresa hay, por lo menos, una restricción, sino fuera así generaría ganancias ilimitadas (Goldratt, 1990). Siendo las restricciones factores que bloquean a la empresa en la obtención de más ganancias, toda gestión que apunte a ese objetivo debe gerenciar focalizando en las restricciones. Lo cierto de que TOC es una metodología sistémica de gestión y mejora de una empresa.

Los cuellos de botella, también se pueden identificar con las barreras a la exportación que varios autores ya han estudiado entre las que se señalan las barreras por falta de conocimiento por parte de los empresarios del proceso exportador, las barreras competitivas por el número de competidores o la falta de capacidad o tecnificación para hacer frente a esta, las barreras gubernamentales falta de apoyo estatal, impuestos, tarifas arancelarias, normatividades y pocos incentivos y barreras culturales propias de la negociación con otras culturas (Escandón, et al., 2015).

En el proceso logístico de las Pymes de confecciones se dan principalmente por la falta de conectividad entre el proceso interno de la empresa y su proceso de exportaciones, falta claridad en los procesos productivos, la falta de planeación y desconocimiento al realizar una exportación, un proceso logístico de distribución deficiente donde Colombia es un país que tiene un desarrollo bajo comparado con su competencia internacional y sus principales clientes Banco Mundial (BM,2016) 
Los efectos de estas restricciones para la logística son en la ventaja competitiva de las empresas en el mercado internacional. La teoría de la ventaja competitiva expuesta por Michael Porter, incluye que las empresas deben tener primero liderazgo por costos, el lograr este liderazgo significa que la firma tiene el costo más bajo en su industria, segundo ser un líder por proximidad o paridad y tercero si no se logra competir por costos porque otra empresa ha asegurado su competencia en costos, no trae buenos resultados (Porter, 1997).

Así mismo, hay que tener en cuenta que la competitividad comercial entendida como "la capacidad de una industria o empresa para producir bienes con patrones de calidad específicos, utilizando más eficientemente recursos que otras empresas o industrias semejantes del resto del mundo durante un cierto período de tiempo" (Haguenauer L, 1989).

De acuerdo a lo anterior, la inserción efectiva de las Pymes en el mercado internacional implica por un lado factores de competitividad internos de las empresas entre ellos los procesos productivos eficientes, políticas administrativas claras y la efectividad logística y por otro lado factores externos como las políticas comerciales y la infraestructura de transportes (Browersox, et al., 2002).

Es por esto, los estados junto con las empresas deben generar políticas para el buen funcionamiento de las exportaciones y trabajar para el mejoramiento de la competitividad de los productos en el mercado nacional y en el mercado internacional

Las actuales condiciones de competitividad frente al mercado internacional son las siguientes: 
Tabla 1. Características de la competencia de Colombia en confecciones:

\begin{tabular}{|c|c|c|c|c|}
\hline Aspectos & Colombia & Europa & China & Estados Unidos \\
\hline $\begin{array}{l}\text { Gestión } \\
\text { tecnológica }\end{array}$ & $\begin{array}{l}\text { Cuenta con la } \\
\text { implementación } \\
\text { de nuevas } \\
\text { técnicas de } \\
\text { confección y } \\
\text { Maquinaria } \\
\text { especializada. }\end{array}$ & $\begin{array}{l}\text { Cuenta con } \\
\text { mecanismos } \\
\text { sofisticados en } \\
\text { maquinaria y } \\
\text { satélites de } \\
\text { confección } \\
\text { especializados. }\end{array}$ & $\begin{array}{l}\text { Mecanismos } \\
\text { sofisticados } \\
\text { en Maquinaria } \\
\text { y satélites de } \\
\text { Confección } \\
\text { especializados }\end{array}$ & $\begin{array}{l}\text { La industria } \\
\text { nacional tiene alta } \\
\text { tecnología en } \\
\text { confección y sus } \\
\text { proveedores } \\
\text { son } \\
\text { especializados }\end{array}$ \\
\hline $\begin{array}{l}\text { Gestión } \\
\text { estratégica }\end{array}$ & $\begin{array}{l}\text { Innovación de } \\
\text { diseños y alta } \\
\text { calidad. }\end{array}$ & $\begin{array}{l}\text { Diseños } \\
\text { exclusivos, } \\
\text { calidad, marcas } \\
\text { ecológicas, y } \\
\text { especialistas en } \\
\text { moda por } \\
\text { temporadas. Alta } \\
\text { capacidad en } \\
\text { comercialización }\end{array}$ & $\begin{array}{l}\text { Precios } \\
\text { competitivos } \\
\text { en el mercado } \\
\text { por bajos } \\
\text { costos de } \\
\text { mano de obra } \\
\text { y eficiencia } \\
\text { productiva }\end{array}$ & $\begin{array}{l}\text { La mayoría de su } \\
\text { ropa es } \\
\text { confeccionada por } \\
\text { países terceros }\end{array}$ \\
\hline $\begin{array}{l}\text { Gestión } \\
\text { comercial }\end{array}$ & $\begin{array}{l}\text { Comercializar } \\
\text { la marca propia } \\
\text { en cadenas } \\
\text { minoristas. }\end{array}$ & $\begin{array}{l}\text { Proyectarse a } \\
\text { nivel mundial } \\
\text { como un icono } \\
\text { de moda } \\
\text { pretencioso } \\
\text { consumidores } \\
\text { de alto nivelo } \\
\text { adquisitivo con } \\
\text { gran impacto } \\
\text { visual y } \\
\text { tecnológico }\end{array}$ & $\begin{array}{l}\text { Comercializ } \\
\text { ación al por } \\
\text { mayor y en } \\
\text { paquete } \\
\text { completo }^{3}\end{array}$ & $\begin{array}{l}\text { Comercialización } \\
\text { en tiendas } \\
\text { especializadas, en } \\
\text { locales } \\
\text { comerciales } \\
\text { propios y en las } \\
\text { cadenas más } \\
\text { Importantes de } \\
\text { ropa en el mundo. }\end{array}$ \\
\hline Marcas & $\begin{array}{l}\text { Tennis y Arturo } \\
\text { Calle }\end{array}$ & $\begin{array}{l}\text { Lacoste } \\
\text { (francesa) Hugo } \\
\text { Boss (Alemana) } \\
\text { Scott \& Soda } \\
\text { (Holandesa) } \\
\text { Burberry Brit } \\
\text { (Inglesa) }\end{array}$ & $\begin{array}{l}\text { Paquete } \\
\text { completo }\end{array}$ & $\begin{array}{l}\text { Armani, Tommy } \\
\text { y Ralph Lauren }\end{array}$ \\
\hline
\end{tabular}

Fuente: Elaboración propia.

\footnotetext{
${ }^{3}$ A diferencia del modelo de subcontratación que comprende corte, costura y acabado, las exportaciones de paquete completo comprenden un modelo más integrado de producción que incluye actividades de pre- producción (sourcing, financiamiento e inspección de tela, marcación de patrones y graduación) y de post- producción (lavandería y acabado, etiquetaje, empaque).
} 
Uno de los competidores más importantes es China, por lo cual se ha identificado y diferenciado por lo siguiente:

Los factores logísticos que se identificaron en las entrevistas realizadas se detallan a continuación:

Figura 5. Factores logísticos de competitividad de China.

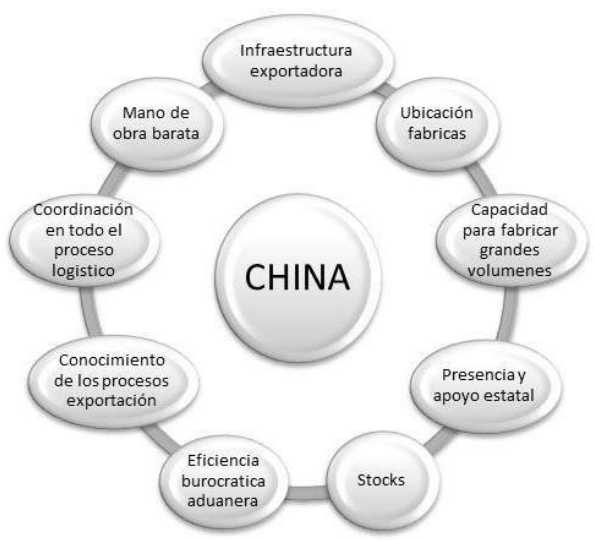

Fuente: Elaboración propia.

Es así como uno de los elementos que se identificaron como restricciones en las exportaciones son el costo de las materias primas (las cuales dependen del precio del algodón) y de la disponibilidad de textiles, de los tiempos y movimientos que tenga la empresa lo cual influye en el uso de la mano de obra y de los recursos de la empresa, de la tecnología de los equipos, del transporte interno, del costo del almacenaje y del transporte internacional

"Jorge Kagwaguchi expone que la gran diferencia está que en China se puede encontrar que el exportador de camisas ya conoce todo el proceso logístico y cuando llega a la aduana de salida de China tiene todos los papeles en regla, son eficientes porque ellos hacen parte de la cadena del negocio, en la cultura asiática todos los procesos de producción tienen nexos claros con las exportaciones. Cuando la mercancía llega a los depósitos fiscales, tienen todos los requerimientos porque tienen esquemas logísticos preparados, y lo 
Análisis de los cuellos de botella en la logística internacional de las Pymes de confecciones en Colombia

hacen desde su producción." (J.Kagwaguchi, comunicación personal 5 de agosto de 2015)

Con respecto a la competitividad de costos:

Tabla 2. Comparativo de costos principales competidores

\begin{tabular}{|c|c|c|c|c|}
\hline Aspectos & Colombia & Europa & China & $\begin{array}{l}\text { Estados } \\
\text { Unidos }\end{array}$ \\
\hline $\begin{array}{l}\text { Valor } \\
\text { Unitario por } \\
\text { camisa en } \\
\text { FOB }\end{array}$ & $\begin{array}{l}\text { Colombia } 40 \\
\text { USD } \\
\text { Goriz } 24 \\
\text { USD }\end{array}$ & $\begin{array}{l}\text { Francia } 108 \\
\text { USD } \\
\text { Italia } 79 \text { USD } \\
\text { Reino Unido } \\
72 \text { USD }\end{array}$ & $\begin{array}{l}\text { China 12 USD } \\
\text { Bangladesh } 9 \\
\text { USD }\end{array}$ & N/A \\
\hline $\begin{array}{l}\text { Costo al } \\
\text { consumidor } \\
\text { final } \\
\text { promedio }\end{array}$ & 45 USD & \begin{tabular}{|l} 
Lacoste 113 \\
USD \\
Hugo Boss \\
145 \\
Scott\& Soda \\
105 \\
Burberry Brit \\
250 \\
\end{tabular} & \begin{tabular}{|l} 
American \\
Eagle 30 USD
\end{tabular} & $\begin{array}{l}\text { Armani } 221 \\
\text { USD } \\
\text { Ralph Lauren } \\
98 \text { USD }\end{array}$ \\
\hline $\begin{array}{l}\text { Reconocimiento } \\
\text { en el mercado }\end{array}$ & Medio & Alto & Alto & Alto \\
\hline
\end{tabular}

Fuente. Elaboración propia.

Michael Porter (1997) resalta que:

"el comportamiento del costo depende de varios factores estructurales que influyen en el costo, las llamadas directrices: economías de escala, aprendizaje, el patrón de capacidad de utilización, eslabones interrelacionales, integración, tiempo, políticas discrecionales, ubicación y factores institucionales". (p.16)

Factores que las empresas deben tener en cuenta al momento de realizar su proceso exportador, de esta manera la logística competitiva busca altos niveles de servicios con costos bajos (Ballesteros y Ballesteros, 2006). Existen modelos de gestión logística que buscan analizar el desempeño de los países, las empresas pymes se caracterizan por un servicio al cliente alto, utilizando la logística adecuada, por lo cual los competidores no podrán mejorar su desempeño (Cano y Orue, 2015). 
Los países requieren de una logística eficaz en exportaciones tiene que ver con la infraestructura y los servicios prestados, de esta manera evitar que se aumenten los costos de exportación.(Guasch, 2011). Es por esta razón que es necesario el mejoramiento de la infraestructura - caminos, puertos, aeropuertos, ferrovías (hardware) y los servicios logísticos -servicios procesos para transportar y comercializar los productos-, trámites aduaneros, licencias, tarifas, inspecciones acceso a instalaciones (software) en las Pymes que son un gran \% en los países latinoamericanos (Guasch, 2011).

\section{Identificación de restricciones logísticas de las pymes de confecciones}

Según un estudio realizado por la CEPAL en el año 2007 a 300 pymes exportadoras de Colombia, Chile y Argentina, las pymes exitosas se caracterizan por: a. El tamaño de la empresa lo cual ayuda en la eficacia y eficiencia de sus operaciones productivas y comerciales, b. El aprendizaje exportador que tienen, lo cual hace que realice ventas más fáciles y el aprendizaje temprano de esta actividad, c. El mejoramiento en sus actividades básicas de las empresas como el perfeccionamiento del producto, normas técnicas y de calidad, mejor servicio al cliente, prácticas de producción y habilidades gerenciales, d. El desarrollo de competencias tecnoorganizacionales, como el tener un departamento de I \&D, inversión en maquinaria y equipo, cambio en sus procesos productivos. e. Competencias endógenas comerciales y de gestión del comercio exterior, cartera de clientes diversificada junto con sus canales de distribución, conocimiento del mercado de destino y desempeño de productos exportados, f. Presencia de elementos macroeconómicos y entorno institucional, debido al carácter sistémico de la competitividad (Milesi, et al., 2007).

Es posible que Colombia tenga algunos cuellos de botella en la logística que le impiden aumentar sus exportaciones e incentivar a las Pymes a crear nuevos ingresos en el mercado exterior. Desde la teoría de las restricciones, la logística que 
Análisis de los cuellos de botella en la logística internacional de las Pymes de confecciones en Colombia

manejan las empresas en su proceso de exportación cuenta con cuellos de botella que han impedido su eficiencia logística y aumente los costos de sus exportaciones.

La causa probable de esta situación es principalmente la mirada global que Colombia ha tenido sobre la logística internacional, sin enfocarse en la particularidad y diversidad que tiene cada uno de los procesos logísticos tanto al interior de la empresa como en el de exportaciones.

\section{Restricciones logísticas al interior de la empresa}

Como se analizó anteriormente los cuellos de botella impiden las ganancias ilimitadas de las empresas por lo tanto es necesario identificarlas desde el punto de vista de los empresarios y de los expertos.

La calificación de los entrevistados con relación a algunos factores en la gestión de la logística interna de la empresa fue la siguiente:

Las empresas entrevistadas calificaron su gestión logística en producción de la siguiente manera, siendo 1 la menor eficiencia y 5 mayor eficiencia: Industrias Goriz calificó su gestión en 1 por su profundo desconocimiento y poco control (V.Gómez, comunicación personal, 5 de noviembre de 2015) y Los Vestidos como 3, por ser una empresa reconocida en el mercado que ha asumido controles para mejorar su eficiencia productiva y responder a los mercados de manera adecuada (M.Giraldo,comunicación personal, 10 de noviembre de 2015)

Los empresarios señalaron una dificultad alta al momento de realizar la compra de materia prima, negociar con los proveedores y procesar los pedidos oportunamente, hay que tener en cuenta que en el proceso productivo, los tiempos y movimientos no son frecuentemente analizados, logrando desperdicios en la empresa. Es por esta razón que según los expertos hay altos grados de dificultad al implementar las herramientas que faciliten el proceso y disminuyan los costos productivos (O. Diaz, comunicación personal, 10 de septiembrede 2015) (O. Solarte, comunicación personal, 15 de septiembre de 2015). Con relación a su capacidad de producción es 


\section{Egna Avendaño Cardenas y Harold Silva Guerra}

Telos Vol. 20, No. 3 (2018). 510-536

fácilmente identificada por los empresarios y el costeo del producto tiene un grado bajo de dificultad, lo que disminuye los riesgos operacionales en las empresas (J. Kagwaguchi, comunicación personal 5 de agosto de 2015).

Por lo tanto, en el área de producción los expertos entrevistados coinciden en que las causas principales de la ineficiencia logística son: la ausencia de procedimientos, la falta de planeación comercial y de producción y la poca estandarización de los procesos (E.Valero, comunicación personal 10 de Octubre de 2015) (M.Medina, comunicación personal 15 de Octubre de 2015).

Es así como los cuellos de botella identificados por los expertos entrevistados son los siguientes:

Figura 6. Cuellos de botella identificados para el proceso de producción.

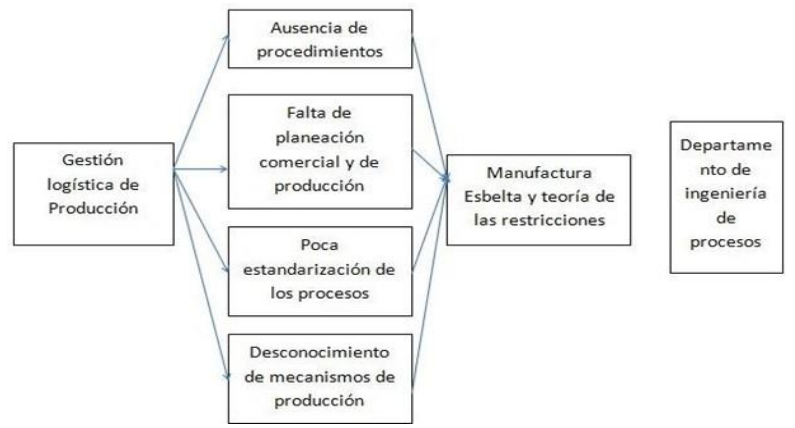

Fuente. Elaboración propia.

De acuerdo al gráfico anterior, para mejorar la eficiencia, el empresario de las Pymes, debe iniciar su proceso de capacitación y de implementación de herramientas como Manufactura Esbelta y de teorías como las de las restricciones que hacen que se identifiquen los elementos logísticos que pueden estar afectando la cadena logística (O. Diaz, comunicación personal, 10 de septiembrede 2015) (O. Solarte, comunicación personal, 15 de septiembre de 2015). Incluyen que "es necesario que la empresa tenga un departamento de ingeniería, especialmente de ingeniería de procesos - lo cual previene que los productos se vuelvan obsoletos prontamente" (M. Medina, comunicación personal 15 de Octubre de 2015). 
Análisis de los cuellos de botella en la logística internacional de las Pymes de confecciones en Colombia

Asimismo, resaltan la importancia de revisar constantemente la planeación de la empresa a todo nivel para diseñar un mejoramiento continuo en los procesos de producción y tener unos procedimientos administrativos claros, donde se diseñen estrategias que evidencien las ventajas y debilidades de la empresa en su gestión logística.

De esta manera, los factores en la logística interna que se deben tener en cuenta para llevar a cabo una exportación exitosa son:

- Capacidad de producción- oferta exportable

- Capacidad financiera de la empresa

- Selección del producto a exportar

- Proceso de producción del producto a exportar

- Identificación y planificación de las etapas para llegar a exportar

- Estrategias para el servicio al cliente

- Gestión de inventarios

- Procesamiento de pedidos

- Gestión eficiente de la cadena de distribución

Teniendo en cuenta lo anterior, la empresa de confecciones debería implementar un mecanismo para controlar los tiempos y movimientos, tener en cuenta su capacidad de producción, conocer sus procesos logísticos, controlar sus inventarios y lograr una distribución eficiente del producto, de esta manera darle un buen servicio al cliente que logre un producto con costos y calidad competitiva

\section{Restricciones logísticas en la logística internacional}

Las restricciones internas en la empresa afectan la competitividad, pero hay otros cuellos de botella que tienen que ver con el proceso exportador que impiden que la empresa pueda llegar al mercado de manera exitosa.

A partir de las entrevistas realizadas, se identificó que en la distribución física de la mercadería, se encuentran aspectos relacionados directamente con el 


\section{Egna Avendaño Cardenas y Harold Silva Guerra}

Telos Vol. 20, No. 3 (2018). 510-536

comercio exterior; entre ellos están la documentación, el empaque y embalaje, el transporte terrestre, las inspecciones, las instalaciones portuarias, el almacenaje y el transporte internacional de la mercadería. Para lo cual algunas actividades son realizadas directamente por el empresario como lo son la documentación, el empaque y el embalaje y las demás actividades o bien se delegan al operador logístico como lo son el transporte o son actividades que realiza el gobierno.

En las entrevistas realizadas a los expertos de exportaciones y empresarios sobre el grado de dificultad al momento de exportar, donde 0 es el menor grado y 5 el mayor grado de dificultad, se obtiene como resultado las siguientes conclusiones:

Los empresarios otorgan el mayor grado de dificultad para exportar al costo del transporte interno y del transporte internacional, el almacenaje y la revisión de aduanas. Las mayores dificultades que afrontan las pymes según los resultados son el almacenaje, la documentación y el costeo, dados principalmente por el poco conocimiento del mercado exterior por parte del empresario y por la falta de cumplimiento de los requerimientos de las mercaderías al momento de exportar. El cuadro separa claramente las actividades internas de la empresa (para las cuales el empresario tiene mayor control y puede disminuir sus restricciones), y las actividades externas (para lo cual son restricciones en su mayoría estatales y de condiciones geográficas).

Además, para Gabriel Quiroga quién se encarga de esta gestión de distribución en Meridian Logistics, plantea los siguientes elementos que influyen en las exportaciones (G. Quiroga, comunicación personal, 20 de septiembre de 2015). 
Análisis de los cuellos de botella en la logística internacional de las Pymes de confecciones en Colombia

Figura 7. Elementos que influyen en las exportaciones.

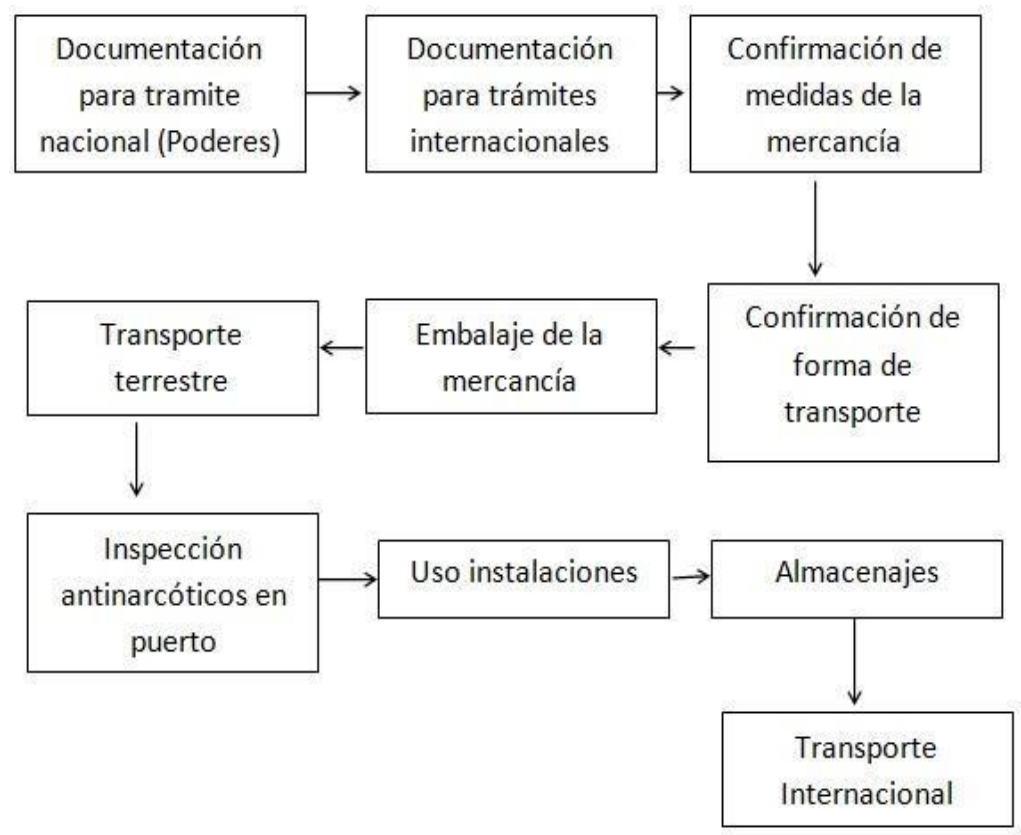

Fuente. Elaboración propia.

Estos procedimientos son básicos en el proceso de exportación, hay que tener en cuenta que el tema de documentación, consolidación de la carga, el medio de transporte a escoger, embalaje, inspección de narcóticos, y almacenajes, son temas que normalmente el empresario delega al Operador logístico y que este hace la coordinación logística para que la mercadería llegue en condiciones óptimas como las espera el exportador, por lo cual pierde control de su mercadería.

De esta manera se señalaron algunos factores logísticos que el empresario debe tener presentes cuando tome la decisión de exportar:

- Selección adecuada del INCOTERM

- Conocimiento del mercado al que se va a exportar

- Diligenciamiento de la documentación necesaria 


\section{Egna Avendaño Cardenas y Harold Silva Guerra}

Telos Vol. 20, No. 3 (2018). 510-536

- Cumplimiento de la reglamentación del país importador

- Análisis de tiempos de tránsito y entrega de mercancía

- Costos de transporte local en punto de llegada

- Selección de transporte internacional

- Manipulación de la carga

- Costos de documentación y tramitología

Marlene Díaz afirmó:

"la necesidad de la empresa en conocer todo su proceso de exportación desde el momento en que se empieza a producir la prenda para el mercado internacional y la ruta que tiene que llegar a su cliente final. Esto es lo básico en el proceso de exportación, es entender el proceso que tiene y antes debe tener conocimiento del mercado y condiciones de accesos, para generar procesos de exportación exitosos" (M. Diaz, comunicación personal, 25 de septiembre de 2015)

El buen manejo de la logística de exportaciones según Marlene Díaz, debe tener como respaldo las capacitaciones para entender los procesos y la adaptación de la empresa a nuevas condiciones del mercado internacional.

"Estas capacitaciones deben enfocarse en diferenciar el lenguaje, los instrumentos, elementos, actores del proceso exportador, con la logística interna de la empresa. Hay que tener en cuenta que la empresa debe tener claro su proceso logístico interno para entender y procesar lo relacionado con la logística externa" (M. Diaz, comunicación personal, 25 de septiembre de 2015)

Oscar Solarte por su parte resalta que el buen manejo depende del “conocimiento de la normatividad y políticas de exportación y de las condiciones de la importación que maneja el país de destino" (O. Solarte, comunicación personal, 15 de septiembre de 2015)

En conclusión, los elementos que se identificaron en las encuestas como restricciones internas de la empresa y del proceso exportador en la industria de confecciones son:

a. El costo de las materias primas (las cuales dependen del precio del algodón). 
Análisis de los cuellos de botella en la logística internacional de las Pymes de confecciones en Colombia

b. La disponibilidad de la materia prima, los textiles.

c. Los tiempos y movimientos en la producción, lo cual influye en el uso de la mano de obra y de los recursos de la empresa.

d. La tecnología de los equipos

e. El costo y seguridad del transporte interno

f. El costo del almacenaje.

g. Desconocimiento de la terminología logística al momento de contactarse con los operadores logísticos.

h. El costo del transporte internacional.

i. La falta de conocimiento en el mercado internacional

De acuerdo a estos elementos se realiza la siguiente tabla donde se identificaron posibles oportunidades para mejorar la competitividad de confecciones

Tabla 3. Cuellos de botella y oportunidades en la logística de producción y de exportaciones de las Pymes de Colombia

\begin{tabular}{|c|c|}
\hline & Cuellos de botella \\
\hline $\begin{array}{l}\text { Logística } \\
\text { empresarial }\end{array}$ & 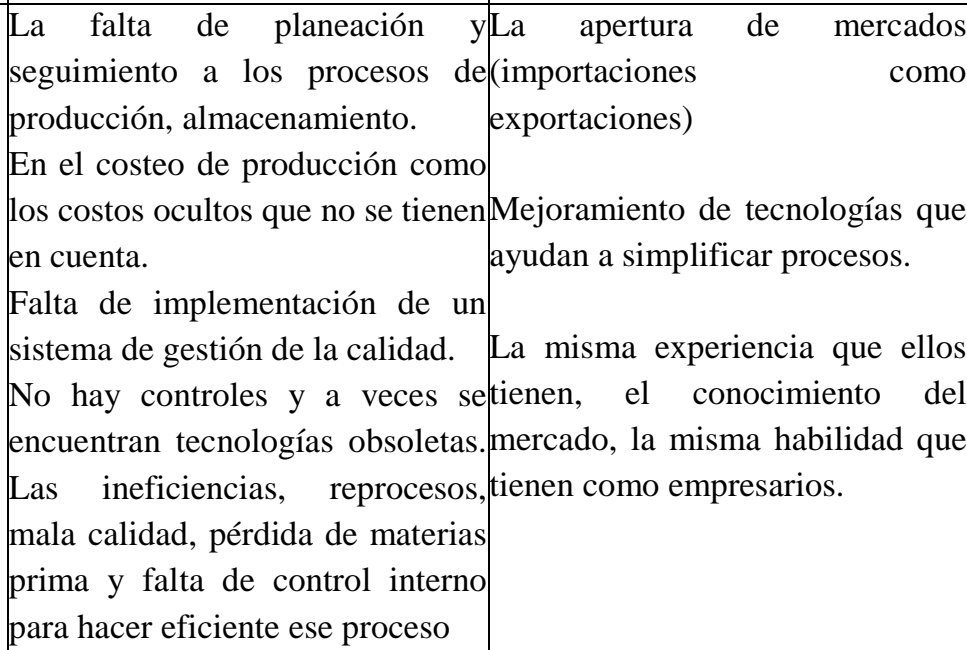 \\
\hline
\end{tabular}




\begin{tabular}{|c|c|c|}
\hline \begin{tabular}{|l|} 
Logística de \\
exportación
\end{tabular} & $\begin{array}{l}\text { La planeación y el seguimiento a } \\
\text { los procesos exportadores. } \\
\text { Atraso en infraestructura nacional } \\
\text { para garantizar medios suficientes } \\
\text { y falta apoyo del gobierno en } \\
\text { subsidios a las empresas. Falta de } \\
\text { conocimiento en el procedimiento } \\
\text { a exportar y } \\
\text { documentación. } \\
\text { Altos costos en trámites }\end{array}$ & $\begin{array}{l}\text { Los tratados de libre comercio y } \\
\text { las buenas } \\
\text { relaciones } \\
\text { internacionales que tiene el país } \\
\text { en este momento. } \\
\text { La calidad de los productos } \\
\text { elaborados igualando los } \\
\text { estándares } \\
\text { ales específicos requeridos para } \\
\text { cada producto. }\end{array}$ \\
\hline
\end{tabular}

Fuente. Resultado de entrevistas realizadas. Elaboración propia.

\section{CONCLUSIONES}

\section{Sector de confecciones}

Las pymes de confecciones de Bogotá deben analizar la posibilidad de orientar sus estrategias comerciales, en especial, más hacia el mercado externo y no tanto al mercado interno, para lograr un mayor grado de expansión y crecimiento de sus utilidades.

La competencia de las pymes de confecciones es la del mercado Chino, el cual se caracteriza por llegar con paquete completo con altos volúmenes y bajos costos, por lo que las empresas de confecciones de Colombia, deben mejorar su competitividad con la diferenciación en diseños exclusivos. Es importante la vinculación de las pymes en el clúster de confecciones para mejorar la eficiencia logística y la disminución de los costos.

\section{Gestión logística empresarial}

Los grados de mayor dificultad en la gestión logística empresarial que se identificaron fueron la relación con los proveedores, el procesamiento de los pedidos, los tiempos y movimientos en el proceso productivo, para lo que la empresa debe generar mejoramientos continuos y así poder disminuir los cuellos de botella. Así 
Análisis de los cuellos de botella en la logística internacional de las Pymes de confecciones en Colombia

mismo para superar sus inconvenientes logísticos, la empresa pyme de confecciones debe diseñar procedimientos de producción claros, donde se tengan en cuenta los tiempos y movimientos; también debe planear su producción y las estrategias de comercialización de sus productos en el mercado de destino, con el fin de maximizar sus ganancias y disminuir sus costos productivos.

La competitividad del costo no solo depende del producto cuando está listo sino cuando llega al cliente final; por lo tanto, los costos de importación deben ser tenidos en cuenta al realizar la exportación y así calcular el valor real de la camisa de hombre.

Los costos que el empresario debe tener en cuenta para mejorar la eficiencia logística de producción son: la mano de obra, proceso productivo, el empaque, el mejoramiento de la tecnología como la compra de maquinaria de etiquetado y el aprovechamiento de capacidad productiva. Hay costos que no dependen del exportador como el costo de la materia prima.

\section{Gestión logística de exportaciones}

Es necesario capacitar al exportador, para que tenga el procedimiento más controlado hasta el consumidor final y así evitar el recargo de costos y cuidar la marca de la empresa.

Los costos que el empresario debe tener en cuenta para mejorar la eficiencia logística de exportación son: las inspecciones antes de la exportación, la coordinación con el operador logístico de la documentación y el cumplimiento de los requisitos exigidos en aduana. Hay costos que no dependen del exportador como el transporte, el almacenaje, las demoras, los procedimientos aduaneros, para lo cual el estado debe tomar las medidas apropiadas para mejorar la eficiencia.

El empresario deberá conocer todo lo relacionado con la exportación, en especial documentación y gestiones que delega normalmente al operador logístico, para mejorar su eficiencia y competitividad en los despachos. 


\section{Referencias bibliográficas}

Acosta, Leidy y Guerrero, Katherine (2005). Evolución de la competitividad de las pymes del sector textil y confecciones Bogotá. Universidad de la Salle. Grado en Economía. Colombia.

Antún, Juan. (2004). Logística internacional. Universidad Autónoma de México. México.

Ballesteros, Diana y Ballesteros, Pedro (2004). La logística competitiva y la administración de la cadena de abastecimiento. Scientia et Technica, Volumen 1. No.21. Colombia (Pp. 201-206).

Banco Mundial. (2016). Trade Logistics in the Global Economy. World Bank. Estados Unidos.

Bloch, Roberto (2011a). Las pequeñas y medianas empresas, la experiencia en Italia y en la Argentina. AD-HOC editores. Argentina.

Bloch, Roberto (2011b). Logística militar y civil. Transporte fluvial.AD-HOC editores. Argentina.

Bloch, Roberto (2015). Entrevista Personal. Buenos Aires, Argentina.

Cámara de Comercio de Bogotá. (2017). Plan estratégico para la región 2007-2019. Obtenido de Cámara de Comercio de Bogotá. Extraído de: http://camara.ccb.org.co/documentos/3528 textiles_y confecciones_peer 20 07 2019.pdf Consulta: 12/11/2017

Cano, Patricia, \& Orue, Fernando. (2015). Modelo de gestión logística para pequeñas.

Contaduría y Administración. Volumen 60 No.1. México (Pp. 181-203)

Carro, Roberto, \& González, Daniel. (2008). Logística empresarial. Editorial Universidad Nacional de Mar de Plata. Argentina.

Departamento Administrativo Nacional de Estadística. (2016). Encuesta Anual Manufacturera -EAM-. Colombia.

Departamento Administrativo Nacional de Estadística. (2016). Estudio comercio exterior de Textil y confecciones. Colombia.

Departamento Nacional de Planeación. (2016). Política nacional de logística. Consejo Nacional de la Política Económica y Social. Colombia

Díaz, Miguel. (2015). Entrevista Personal. Bogotá, Colombia.

Escandón, Diana, Hurtado, Andrea y Castillo, Maribel. (2013). Influencia de las barreras a la exportación sobre el compromiso exportador y su incidencia en los resultados internacionales. Escuela de Administración y Negocios. Volumen 1. No 75. Colombia (Pp. 38-55).

Giraldo, Carlos (2015). Entrevista Personal. Bogotá, Colombia.

Goldratt, Eliyahu (1990). The Haystack Syndrome: Siffing Information Out the data Ocean. North River Press. Estados Unidos.

Goldratt, Eliyahu. (1994). What is this Thing Called the Theory of Constraints, and How should it be Implemented? North River Press. Estados Unidos. 
Análisis de los cuellos de botella en la logística internacional de las Pymes de confecciones en Colombia

Goméz, Martha., \& Acevedo, José. (2000). Logística de aprovisionamiento. Corporación Jhon F. Kennedy. México.

Gómez, Val. (2015). Entrevista Personal. Bogotá, Colombia.

Guasch, Judith. (2011). La logística como motor de competividad en América Latina y el Caribe. V Foro de Competitividad de las Américas para el Banco Interamericano de Desarrollo y el Compete Caribbean. Santo Domingo, República Dominicana.

Haguenauer, Lia. (1989). Competitividade, conceitos e medidas. Uma resenha da bibliografía recente comêm fase no caso brasileiro. Universidad Federal de Rio de Janeiro. Brasil

Kagwaguchi, Jea (2015). Entrevista Personal. Buenos Aires, Argentina.

Long, Douglas (2006). Logística internacional. Cadena de abastecimiento... Noriega editores. México.

López, Gary (2015). Entrevista Personal. Bogotá, Colombia Medina, Juan (2015). Entrevista Personal. Bogotá, Colombia

Milesi, Dario, Moori, Virginia, \& Veronica, Robert (2004). Desarrollo de ventajas competitivas: Pymes exportadoras exitosas. Colombia, Chile y Argentina.

Revista de la CEPAL. No 92. Argentina (Pp. 25-43).

Montoya, Alexandra, \& Montoya, Ivan (2010). Situación de la competitividad de las Pyme en Colombia: elementos actuales y retos. Agronomía Colombiana. Universidad nacional. Volumen 28. No 1. Colombia (Pp. 107-117).

Porter, Michael (1997). Ventaja competitiva: Creación y sostenimiento de un desempeño superior. Compañía editorial continental, S.A. México

Quiroga, Gilberto. (2015). Entrevista Personal. Bogotá, Colombia

Solarte, Oscar (2015). Entrevista Personal. Bogotá, Colombia

Superintendencia de Sociedades. (2016). Informe de desempeño del sector textil y confecciones. Bogotá: Superintendencia de Sociedades. Colombia

Valero, E. (2015). Entrevista Personal. Bogotá, Colombia.

Zaninovich, Dimitri (2014). Implementación de la Política Nacional de Logística. Departamento Nacional de Planeación-DNP-. Colombia. 